\title{
The effect of input modality on short-term serial recall'
}

\author{
MICHAEL C. CORBALLIS ${ }^{2}$ AND THELMA LOVELESS 3
}

Immediate recall of eight visually presented items was better if the first four were presented fast and the last four slowly(FS Condition) than if the first four were slow and the last four fast (SF Condition), supporting an hypothesis that Ss tend to rehearse cumulatively. Using aural presentation, Schonfield \& Donaldson (1966) have obtained the opposite result, supporting decay theory. These and other results suggest that rehearsal of serially presented information is more efficient and flexible when presentation is visual than when it is aural.

Schonfield \& Donaldson (1966) have reported that Ss were better able to recall series of eight digits if the first four were presented slowly and the last four fast (SF Condition) than if the first four were presented fast and the last four slowly (FS Condition). Because, under the FS Condition, each digit except the first and the last must be held in storage slightly longer than the corresponding digit under the SF Condition, the authors interpreted this result as evidence that autonomous decay is a factor in forgetting over short intervals.

Using a similar technique, Corballis (1966) obtained a contradictory result. Series of nine digits were presented under two conditions; in Condition $D$ the intervals between digit onsets were initially long but gradually decreased within series, while in Condition I the intervals were initially short but gradually increased within series. Recall was generally better under Condition I than under Condition D. This result is the opposite to that predicted by the hypothesis of autonomous decay. Rather, it supports a hypotheesis that Ss rehearse cumulatively; that is, they rehearse more digits during later stages of presentation that during early stages. Consequently, rehearsal is more effective under Condition I because the longer intervals occur later in the series.

The discrepancy between the results of these two studies may be partly a function of presentation modality. In the former, the digits were presented aurally. In the latter, presentation was both aural and visual, but the difference between Conditions I and $\mathrm{D}$ was much more pronounced when presentation was visual than when it was aural. Indeed, there was little evidence that rehearsal was cumulative when presentation was aural. It was argued that cumulative rehearsal is characteristic of memory processing of visually presented information, but is probably not characteristic of processing of auditory information.

The purpose of the present experiment was to compare immediate recall under the SF Condition with that under the FS Condition, using visual instead of aural presentation. The temporal characteristics of these two conditions were approximately the same as in Schonfield and Donaldson's study. The cumulative rehearsal hypothesis would lead us to expect better recall under the FS Condition, contrary to Schonfield and Donaldson's result.

\section{Method}

Ss were 10 men and 10 women from an introductory psychology class.

Digits were projected by means of a multiplestimulus projector, designed to project the digits 0 through 9, or a red flash, onto a screen measuring $1-1 / 2$ in. $x 2$ in. Output from a pre-programmed multichannel tape recorder controlled selection and timing of the digit presentations. Digits were presented in series of eight, randomly selected except that no digit could appear twice in the same series. Each digit was presented for $0.3 \mathrm{sec} .$, and the intervals between successsive digit onsets were as follows: FS Condition: $0.6,0.6,0.6,2.1,2.1,2.1$, and $2.1 \mathrm{sec}$.; SF Condition: $2.1,2.1,2.1,2.1,0.6,0.6$, and $0.6 \mathrm{sec}$. Each series was preceded by a red flash, which served as a warning signal, and followed $0.6 \mathrm{sec}$. after offset of the last digit by another red flash, as a signal to respond.

Ss were tested individually. They were instructed to report the digits, in the order in which they had been presented, as soon as the red flash appeared following presentation. An interval of 10 sec. was allowed between trials, for $S$ to respond. His responses were recorded on tape.

Each $S$ received 12 trials under each condition; half the Ss received the 12 trials under the FS Condition first, followed by those under the SF Condition. The other half received the SF Condition followed by the FS Condition. Each $S$ was given a practice trial at a constant presentation rate before the experimental trials began.

\section{Results and Oiscussion}

The average number of series correctly recalled, out of 12, was 5.20 under the FS Condition and 3.95 under the SF Condition; a series was considered correctly recalled only if all eight digits were reported in the correct order. Analysis of variance, Latin Square design, indicated that this difference was significant $(p<.05$, one-tailed).

Comparison of this result with that of Schonfield and Donaldson's study provides further evidence that 
presentation modality is an important variable determining the effect of intra-series variation on immediate recall. For visual presentation the results tend to support the cumulative rehearsal hypothesis; for aural presentation they do not, and may even go in the opposite direction, 28 in Schonfield and Donaldson's study.

Modality also appears to be an important variable in experiments in which presentation rate is held constant within series, but is varied between series. Mackworth (1964) has observed that experiments showing better recall with faster presentation rate, in support of the theory of autonomous decay, have all used aural presentation. Experiments using visual presentation generally show better recall the slower the presentation rate, contrary to decay theory.

It is perhaps paradoxical that experiments involving short-term recall of serially presented items have tended to support decay theory when presentation is aural but not when it is visual, since there are indications that storage of visual information is subject to more rapid decay than is storage of auditory information (e.g., Mackworth, 1964; Murdock, 1966). It is plausible to suppose, however, that rehearsal is more effective when presentation is visual than when it is aural. For aural presentation there may be intramodal interference between the auditory stimulus traces and the traces resulting from S's own verbal rehearsal; consequently $S$ may be obliged to discontinue rehearsal of earlier items while another item is being presented in order to avoid confusion. With this restriction on rehearsal, subsequent recall would be contingent upon decay rather than upon rehearsal. For visual presentation, by contrast, $S$ may be able to maintain items in visual storage while rehearsing earlier items without confusion (cf., Turvey, 1966), so that more flexible rehearsal is possible.

It is clear that rehearsal does not merely prevent decay, as Brown (1958) has implied, but that it can actually enhance recall, especially when presentation is visual. Tulving (1966) has shown that recall is not improved by mere repetition of items, however, but rather that $S$ must impose some "subjective organization" upon the series. This is consistent with our view that effective rehearsal is cumulative, since to impose subjective organization upon a temporal sequence implies integration of earlier items with later items.

\section{References}

Brown, J. Some tests of the decay theory of immediate recall. Quart. J. exp. Psychol., 1958, 10, 12-21.

Corballis, M. C. Rehearsal and decay in immediate recall of visually and aurally presented items. Canad. J. Psychol., 1966, 20, 43-51.

Mackworth, J. F. Auditory short-term memory. Canad. J. Psychol., $1964,18,292-303$.

Murdock, B. B. Visual and auditory stores in short-term memory. Quart. J. exp. Psychol., 1966, 18, 206-211.

Schonfield, D., \& Donaldson, W. Immediate memory as a function of intra-series variation. Canad. J. Psychol., 1966, 20, 218-227.

Tulving, E. Subjective organization and effects of repetition in multi-trial free-recall learning. J. verbal Learn. verbal Behav., $1966,5,193-197$.

Turvey, M. T. The effects of rehearsing analyzed information upon the retrieval of unanalyzed information. Psychon. Sci., 1966, 365-366.

\section{Hotes}

1. Supported by a grant (No. 9425-10) from the Defence Research Board of Canada to Dr. Dalbir Bindra.

2. Now at University of Auckland, New Zealand.

3. Now at Department of Psychological Medicine, Southern General Hospital, Glasgow, Scotland. 